\title{
RAYLEIGH WAVE SCATTERING AT THE FOOT OF A MOUNTAIN
}

\author{
P.S. DESHWAL and K.K. MANN \\ Department of Mathematics \\ Maharshi Dayanand University \\ Rohtak-124001, INDIA \\ (Received March 3, 1986)
}

\begin{abstract}
A theoretical study of scattering of seismic waves at the foot of a mountain is discussed here. A mountain of an arbitrary shape and of width $a(0 \leq x \leq a, z=0)$ in the surface of an elastic solid medium $(z \geq 0)$ is hit by a Rayleigh wave. The method of solution is the technique of Wiener and Hopf. The reflected, transmitted and scattered waves are obtained by inversion of Fourier transforms. The scattered waves behave as decaying cylindrical waves at distant points and have a large amplitude near the foot of the mountain. The transmitted wave decreases exponentially as its distance from the other end of the mountain increases.
\end{abstract}

KEY WORDS AND PHRASES. Rayleigh surface waves, wave motions in elastic solids, reflected and transmitted waves.

1980 AMS SUBJECT CLASSIFICATION CODES. 73D20, 73D10.

1. INTRODUCTION.

Earthquakes throw a grave challenge to human beings. Seismic waves cause the havoc because of their scattering due to the inhomogeneities and discontinuities in the surface of the earth. Rayleigh waves, appearing on the surface of the earth, are responsible for destruction of the buildings and loss of human lives. Scattering of seismic waves due to irregularities in the surface leads to large amplification and variation in ground motion during earthquakes.

The paper presents a mechanism by which the energy of a body wave is lessened by partial conversion into reflected body and surface waves and by scattering at the foot of a mountain.

Love wave propagation in case of a surface layer on a solid halfspace has been studied by Sato (1961) using the Wiener-Hopf technique. Kazi (1978) has solved the same problem by an approximate method. Both of them have taken the surface discontinuity along half of the solid halfspace which helps in Fourier transformation and in simplifying mathematical calculations. Scattering of a compressional wave due to the presence of a rigid barrier in the surface of a liquid halfspace has been discussed by Deshwal (1971). He (1981) has also studied the problem of diffraction of a compressional wave when there is a rigid finite strip in the surface of the liquid halfspace. But the 
medium is a liquid halfspace which reduces the number of elastic parameters, the basic equations and the boundary conditions. Not much theoretical study is available on the problem of scattering of elastic waves due to an irregular boundary of finite dimensions. Recently Momoi $(1980,82)$ has studied the problem of scattering of Rayleigh waves by semicircular and rectangular discontinuities in the surface of a solid halfspace. The solutions are not exact because of the approximations used in the solution of these problems.

We propose to discuss here the problem of scattering of Rayleigh waves at the foot of a mountain with its base occupying the region $0 \leq x \leq a, z=0$ in the surface of a solid halfspace $z \geq 0$. Since we are interested in scattering of waves at the foot of a mountain, its shape is immaterial and it is assumed to be rigid such that there is no displacement across the mountain. The method of solution is the Fourier transformation of the basic equations and determination of unknown functions by the technique of Wiener and Hopf (Noble, (1958)).

2. STATEMENT OF THE PROBLEM.

The problem is two-dimensional in $\mathrm{zx}$-plane. The mountain of width a has its base along $0 \leq x \leq a, z=0$ in the surface of an elastic solid halfspace $z \geq 0$ (figure 1). The medium is homogeneous, isotropic and slightly dissipative. If the retarding force of the medium is proportional to the velocity, then the wave equation is

$$
\frac{\partial^{2} \bar{\phi}}{\partial x^{2}}+\frac{\partial^{2} \bar{\phi}}{\partial z^{2}}=\frac{1}{c^{2}} \frac{\partial^{2} \bar{\phi}}{\partial t^{2}}+\frac{\varepsilon}{c^{2}} \frac{\partial \bar{\phi}}{\partial t}
$$

where $c$ is the velocity of propagation and $\varepsilon>0$ is the damping constant. The potential function harmonic in time is

$$
\bar{\phi}(x, z, t)=\bar{\phi}(x, z) \exp (-i \omega t)
$$

The equation $(2.1)$ is now

$$
\left(\nabla^{2}+(\bar{K})^{2}\right) \phi(x, z)=0
$$

where $\bar{K}=\sqrt{\left(\omega^{2}+i \varepsilon \omega\right) / c}=\bar{K}_{1}+i \bar{K}_{2}$ is complex whose imaginary part is small and positive. An incident wave

$$
\begin{aligned}
& \phi_{i}(x, z)=D\left(2 p_{0}^{2}-k^{2}\right) e^{i p_{0} x} e^{-\beta} 0^{z} \\
& \dot{\psi}_{1}(\mathrm{x}, \mathrm{z})=\mathrm{D}\left(21 \mathrm{p}_{0} \beta_{0}\right) \mathrm{e}^{i \mathrm{p}_{0} \mathrm{x}} \mathrm{e}^{-\delta} \delta^{z}
\end{aligned}
$$

where $\mathrm{p}_{0}$ is a root of the Rayleigh frequency equation

$$
f(p)=\left(2 p^{2}-k^{2}\right)-4 p^{2} \beta \delta=0, \beta=\sqrt{\left(p^{2}-(\bar{k})^{2}\right)}, \delta=\sqrt{\left(p^{2}-k^{2}\right)}
$$

$B_{0}=B\left(\mathrm{P}_{0}\right), \quad \delta_{0}=\delta\left(\mathrm{p}_{0}\right)$, strikes at the foot of the mountain from the region $x<0$.

The potential $\psi(x, z)$ satisfies the wave equation

$$
\left(\nabla^{2}+k^{2}\right) \Psi(x, z)=0
$$

Let the total potentials be

$$
\begin{aligned}
& \phi_{t}(x, z)=\phi(x, z)+\phi_{i}(x, z) \\
& \psi_{t}(x, z)=\psi(x, z)+\psi_{i}(x, z)
\end{aligned}
$$


The potentials are connected with the displacements $(u, w)$ by

$$
u=\partial \phi_{t} / \partial x+\partial \psi_{t} / \partial z, \quad w=\partial \phi_{t} / \partial z-\partial \psi_{t} / \partial z
$$

We assume that, for given $z, \phi(x, z)$ and $\psi(x, z)$ have the behaviour $\exp (-d|x|)$ as $|x| \rightarrow \infty, d>0$. The Fourier transform

$$
\bar{\phi}_{-}(\mathrm{p}, \mathrm{z})=\int_{-\infty}^{0} \phi(\mathrm{x}, \mathrm{z}) \mathrm{e}^{i \mathrm{px}} \mathrm{dx}, \quad \mathrm{p}=\alpha+i \alpha_{0}
$$

is analytic in the region $\alpha_{0}<d$ of the complex p-plane. The Fourier transform

$$
\begin{aligned}
\bar{\phi}(p, z) & =\int_{-\infty}^{\infty} \phi(x, z) e^{i p x} d x \\
& =\int_{-\infty}^{0} \phi(x, z) e^{i p x} d x+\int_{0}^{a} \phi(x, z) e^{i p x} d x+\int_{a}^{\infty} \phi(x, z) e^{i p x} d x \\
& =\bar{\phi}_{-}(p, z)+\bar{\phi}_{a}(p, z)+\bar{\phi}_{+a}(p, z)
\end{aligned}
$$

and its derivatives w.r.t.z are analytic in the strip $-d<\alpha_{0}<d$ of the complex plane. The transform of $\psi(x, z)$ has the same behaviour.

3. BOUNDARY CONDITIONS.

The boundary conditions of the problem are

$$
u=\partial \phi_{t} / \partial x+\partial \psi_{t} / \partial z=0, \quad z=0, \quad 0 \leq x \leq a,
$$

$$
w=\partial \phi_{t} / \partial z-\partial \psi_{t} / \partial x=0, \quad z=0, \quad 0 \leq x \leq a,
$$

(iii) $2 \frac{\partial}{\partial x}\left(\frac{\partial \phi}{\partial z}-\frac{\partial \psi}{\partial x}\right)-k^{2} \psi=0, \quad z=0, \quad x \leq 0, \quad x \geq a$,

(iv) $2 \frac{\partial}{\partial x}\left(\frac{\partial \phi}{\partial x}+\frac{\partial \psi}{\partial z}\right)+k^{2} \phi=0, \quad z=0, \quad x \leq 0, \quad x \geq a$,

The conditions (3.1) and (3.2) subject to (2.3) and (2.7) reduce to

$$
\begin{aligned}
& \phi=-\phi_{i}=-D\left(2 p_{0}^{2}-k^{2}\right) e^{i p_{0} x} e^{-\beta} 0^{z}, \quad z=0, \quad 0 \leq x \leq a, \\
& \psi=-\psi_{i}=-D\left(2 i p_{0} \beta_{0}\right) e^{i p_{0} x} e^{-\delta} z \quad, \quad z=0, \quad 0 \leq x \leq a,
\end{aligned}
$$

4. SOLUTION OF THE PROBLEM.

Fourier transforms of the wave equations (2.3) and (2.7) lead to

$$
\begin{array}{ll}
\mathrm{d}^{2} \bar{\phi} / \mathrm{d} \mathrm{z}^{2}-\beta^{2} \bar{\phi}=0, & \beta= \pm \sqrt{\left(\mathrm{p}^{2}-(\overline{\mathrm{k}})^{2}\right)} \\
\mathrm{d}^{2} \bar{\psi} / \mathrm{d} \mathrm{z}^{2}-\delta^{2} \bar{\psi}=0, & \delta= \pm \sqrt{\left(\mathrm{p}^{2}-\mathrm{k}^{2}\right)}
\end{array}
$$

Since $\phi(x, z)$ and $\psi(x, z)$ are bounded as $z$ tends to infinity and their transforms are also bounded. The solutions of $(4.1)$ and $(4.2)$ are

$$
\begin{aligned}
& \bar{\phi}(p, z)=A(p) e^{-\beta z} \\
& \bar{\psi}(p, z)=B(p) e^{-\delta z}
\end{aligned}
$$

The signs in radicals for $\beta$ and $\delta$ are such that their real parts are positive for all p. We use the notations $\bar{\phi}(p), \bar{\psi}(p)$ for $\bar{\phi}(p, 0), \bar{\psi}(p, 0)$ etc. From (4.3) we obtain

$$
\bar{\phi}^{\prime}(p) / \beta=-\bar{\phi}(p)
$$


This equation is decomposed

$$
\begin{aligned}
& \frac{1}{\sqrt{p-\bar{k}}}\left[\frac{\bar{\phi}_{+}^{\prime}(p)}{\sqrt{p+k}}-\frac{\bar{\phi}_{+}^{\prime}(-\bar{k})}{\sqrt{2 \bar{k}}}\right]+\bar{\phi}_{+}(p)+\frac{\bar{\phi}_{-}^{\prime}(-\bar{k})}{\sqrt{-2 \bar{k}(p+\bar{k})}} \\
= & -\bar{\phi}_{-}(p)-\frac{1}{\sqrt{p+\bar{k}}}\left[\frac{\bar{\phi}_{-}^{\prime}(p)}{\sqrt{p-\bar{k}}}-\frac{\bar{\phi}_{-}^{\prime}(-\bar{k})}{\sqrt{-2 \bar{k}}}\right]-\frac{\bar{\phi}_{+}^{\prime}(\bar{k})}{\sqrt{2 \bar{k}(p-\bar{k})}}
\end{aligned}
$$

The left hand member of $(4.6)$ is analytic in $\alpha_{0}>-d$ and the right hand member in $\alpha_{0}>\mathrm{d}$. They represent an entire function. Further each member tends to zero as $|p| \rightarrow \infty$. By Liouville's theorem, the entire function is identically zero. Equating each member to zero, it is found that

$$
\begin{aligned}
& \bar{\phi}_{+}(p)=-\bar{\phi}_{+}^{\prime}(p) / \beta+\bar{q} F(p), \quad \bar{q}=\bar{\phi}_{+}^{\prime}(\bar{k})=\bar{\phi}_{-}^{\prime}(-\bar{k}) \\
& \bar{\phi}_{-}(p)=-\bar{\phi}_{-}^{\prime}(p) / \beta-\bar{q} F(p), \quad-\bar{q}=\bar{\phi}_{-}^{\prime}(k)=\bar{\phi}_{+}^{\prime}(-k) \\
& F(p)=\frac{1}{\sqrt{2 \bar{k}(p-\bar{k})}}-\frac{1}{\sqrt{-2 \bar{k}(p+\bar{k})}}
\end{aligned}
$$

Similarly, we find from (4.4) that

$$
\begin{aligned}
& \bar{\psi}_{+}(p)=-\bar{\psi}_{+}^{\prime}(p) / \delta+q G(p), \quad q=\bar{\psi}_{+}^{\prime}(k)=\bar{\psi}_{-}^{\prime}(-k) \\
& \bar{\psi}_{-}(p)=-\bar{\psi}_{-}^{\prime}(p) / \delta-q G(p), \quad-q=\bar{\psi}_{-}^{\prime}(k)=\bar{\psi}_{+}^{\prime}(-k) \\
& G(p)=\frac{1}{\sqrt{2 k(p-k)}}-\frac{1}{\sqrt{-2 k(p+k)}}
\end{aligned}
$$

Fourier transforms of (3.5) and (3.6) lead to

$$
\begin{aligned}
& \bar{\phi}_{a}(p)=-D\left(2 p_{0}^{2}-k^{2}\right) \int_{0}^{a} e^{i\left(p+p_{0}\right) x} d x=-\frac{D\left(2 p_{0}^{2}-k^{2}\right)}{i\left(p+p_{0}\right)}\left(e^{i\left(p+p_{0}\right) a}-1\right) \\
& \bar{\psi}_{a}(p)=-D\left(2 i p_{0} \beta_{0}\right) \int_{0}^{a} e^{i\left(p+p_{0}\right) x} d x=-\frac{-2 p_{0} \beta_{0} D}{p^{+} p_{0}}\left(e^{i\left(p+p_{0}\right) a}-1\right)
\end{aligned}
$$

And those of (3.1) and (3.2) result in

$$
\begin{aligned}
& \bar{\psi}_{a}^{\prime}(\mathrm{p})=2 \mathrm{Dp}_{0} \beta_{0} \delta_{0}\left(\mathrm{e}^{i\left(\mathrm{p}+\mathrm{p}_{0}\right) \mathrm{a}}-1\right) /\left(\mathrm{p}+\mathrm{p}_{0}\right) \\
& \bar{\phi}_{\mathrm{a}}^{\prime}(\mathrm{p})=-1 \mathrm{D} \beta_{0}\left(2 \mathrm{p}_{0}^{2}-\mathrm{k}^{2}\right)\left(\mathrm{e}^{1\left(\mathrm{p}+\mathrm{p}_{0}\right) \mathrm{a}}-1\right) /\left(\mathrm{p}+\mathrm{p}_{0}\right)
\end{aligned}
$$

We now multiply (3.3) by $\exp (\mathrm{ipx})$ and integrate between $x=-\infty$ and $x=0$ to find out

$$
2 \int_{-\infty}^{0} \frac{\partial}{\partial x}\left(\frac{\partial \phi}{\partial z}-\frac{\partial \psi}{\partial x}\right) e^{i p x} d x-k^{2} \int_{-\infty}^{0} \psi e^{i p x} d x=0
$$

Since $w=\partial \phi / \partial z-\partial \psi / \partial x$ is continuous, the first integral in (4.17) is integrated by parts to obtain

$$
-2 i p \int_{-\infty}^{0}\left(\frac{\partial \phi}{\partial z}-\frac{\partial \psi}{\partial x}\right) e^{i p x} d x-k^{2} \bar{\psi}_{-}(p)=-2\left(\frac{\partial \phi}{\partial z}-\frac{\partial \psi}{\partial x}\right)_{0}
$$

or

$$
-2 i p \bar{\phi}_{-}^{\prime}(p)+\left(2 p^{2}-k^{2}\right) \bar{\psi}_{-}(p)=\left(\frac{\partial \phi_{1}}{\partial z}-\frac{\partial \psi_{1}}{\partial x}\right)_{0}+2 i p\left(\psi_{1}\right)_{0}=2 \beta_{0} D\left(k^{2}-2 p p o\right.
$$


In the same way, (3.4) leads to

$$
\left(2 \mathrm{p}^{2}-\mathrm{k}^{2}\right) \bar{\phi}_{-}(\mathrm{p})+2 i \mathrm{p} \bar{\psi}_{-}^{\prime}(\mathrm{p})=2 \mathrm{ip}\left[\left(\mathrm{p}-\mathrm{p}_{0}\right)\left(2 \mathrm{p}_{0}^{2}-\mathrm{k}^{2}\right)+2 \mathrm{p}_{0}{ }_{0} \delta_{0}\right]
$$

Solving $(4.8),(4.11),(4.18)$ and $(4.19)$ for $\bar{\phi}_{-}(p)$ and $\bar{\psi}_{-}(p)$, we find

$$
\begin{aligned}
f(p) \bar{\phi}_{-}(p)= & 2 i p \delta\left[q\left(2 p^{2}-k^{2}\right) G(p)-2 i p \beta \bar{q} F(p)\right] \\
+ & 2 i D\left(2 p^{2}-k^{2}\right)\left[\left(p-p_{0}\right)\left(2 p_{0}^{2}-k^{2}\right)+2 p_{0} \beta_{0} \delta_{0}\right]+4 i p \beta_{0} \delta D\left(k^{2}-2 p_{0}\right) \\
f(p) \bar{\psi}_{-}(p)= & \left(2 p^{2}-k^{2}\right)\left[-2 i p \beta \bar{q} F(p)+2 \beta_{0} D\left(k^{2}-2 p_{0}\right)\right] \\
& -2 i p \beta\left[2 i p \delta q G(p)+2 i D\left[\left(p-p_{0}\right)\left(2 p_{0}^{2}-k^{2}\right)+2 p_{0} \beta_{0} \delta_{0}\right]\right]
\end{aligned}
$$

Integrating (3.3) and (3.4) between $x=a$ and $x=\infty$ after multiplying it by $\exp (i p x)$, it is obtained that

$$
\begin{gathered}
\left(2 p^{2}-k^{2}\right) \bar{\psi}_{+a}(p)-2 i p \bar{\phi}_{+a}^{\prime}(p)=-2 \beta_{0} D\left(k^{2}-2 p_{0}\right) e^{i\left(p+p_{0}\right) a} \\
\left(2 p^{2}-k^{2}\right) \bar{\phi}_{+a}(p)+2 i p \bar{\psi}_{+a}^{\prime}(p)=-2 i D\left[\left(p-p_{0}\right)\left(2 p_{0}^{2}-k^{2}\right)+2 p_{0} \beta_{0} \delta_{0}\right] \cdot \\
\exp \left(i\left(p+p_{0}\right) a\right)
\end{gathered}
$$

Inversion of Fourier transforms will give various waves.

5. VARIOUS WAVES.

The factor $\exp (-i p x)=\exp (-i \alpha x) \exp \left(\alpha_{0} x\right)$ in the inverse transforms makes the integrals vanish at infinity in the upper part of the complex plane if $x<0$ and in the lower part if $x>0$. For waves in the region $x<0$, we have

$$
\phi(x, z)=\frac{1}{2 \pi} \int_{-\infty+i \alpha_{0}}^{\infty+i \alpha_{0}} \bar{\phi}_{-}(p, z) e^{-i p x} d p
$$

where the line integral (5.1) is in the strip $-d<\alpha_{0}<d$. The contour is in the upper half of the complex plane where $\bar{\phi}_{-}(-p, z)$ is analytic and hence

$$
0=\frac{1}{2 \pi} \int_{-\infty+i \alpha_{0}}^{\infty+i \alpha_{0}} \bar{\phi}_{-}(-p, z) e^{-i p x} d p
$$

From (5.1) and (5.2), it is found that

$$
\phi(x, z)=\frac{1}{2 \pi} \int_{-\infty+i \alpha_{0}}^{\infty+i \alpha_{0}}\left[\bar{\phi}_{-}(p, z)-\bar{\phi}_{-}(-p, z)\right] e^{-i p x_{d}}
$$

To find the integrand, the wave equation (2.3) is integrated from $x=\infty$ to $x=0$ after multiplying it by $\exp (i p x)$, to find out

$$
\mathrm{d}^{2} \bar{\phi}_{-} / \mathrm{dz} \mathrm{z}^{2}-\beta^{2} \bar{\phi}_{-}=-(\partial \phi / \partial \mathrm{x})_{0}+1 \mathrm{p}(\phi)_{0}
$$

Changing $p$ to $-p$ and subtracting the resulting equation from (5.4), we obtain

$$
\left(d^{2} / d z^{2}-\beta^{2}\right)\left[\bar{\phi}_{-}(p, z)-\bar{\phi}_{-}(-p, z)\right]=-2 i p D\left(2 p_{0}^{2}-k^{2}\right) \exp \left(-\beta_{0} z\right)
$$

whose complete solution is

$$
\bar{\phi}_{-}(p, z)-\bar{\phi}_{-}(-p, z)=E(p) e^{\beta z}+E_{1}(p) e^{-\beta z}+\frac{2 i p D\left(2 p_{0}^{2}-k^{2}\right) e^{-\beta} 0^{z}}{p^{2}-p_{0}^{2}}
$$

To find $E$ and $E_{1}$, we take $z=0$ in (5.6) and use (4.19), it is found that 


$$
\begin{aligned}
\bar{\phi}_{-}(p, z)-\bar{\phi}_{-}(-p, z)= & 2 \operatorname{Esinh} \beta z+\frac{4 i p}{f(p)}\left[\left(2 p^{2}-k^{2}\right) \delta q G(p)\right. \\
& \left.+D\left(2 p^{2}-k^{2}\right)\left(2 p_{0}^{2}-k^{2}\right)+2 D \beta k_{0} k^{2} \delta\right] e^{-\beta z} \\
& +2 i p D\left(2 p_{0}^{2}-k^{2}\right)\left(e^{-\beta}{ }^{z}-e^{-\beta z}\right) /\left(p^{2}-p_{0}^{2}\right)
\end{aligned}
$$

Differentiating (5.7) w.r.t. $z$ and putting $z=0$, we find

$$
\begin{aligned}
\bar{\phi}_{-}^{\prime}(p)-\bar{\phi}_{-}^{\prime}(-p) & =2 B E-\frac{4 i p \beta}{f(p)}\left[\left(2 p^{2}-k^{2}\right) \delta q G(p)+2 D \beta k_{0} k^{2} \delta\right. \\
& \left.+D\left(2 p^{2}-k^{2}\right)\left(2 p_{0}^{2}-k^{2}\right)\right]+\frac{2 i p D\left(B-\beta_{0}\right)\left(2 p_{0}^{2}-k^{2}\right)}{p^{2}-p_{0}^{2}}
\end{aligned}
$$

$2 \mathrm{E}$ is obtained from here as the left hand member is known from (4.8) and (4.20). We find the integrand in (5.3) to be

$$
\begin{aligned}
\bar{\phi}_{-}(p, z)-\bar{\phi}_{-}(-p, z) & =\frac{2 i p D\left(\beta-\beta_{0}\right)\left(2 p_{0}^{2}-k^{2}\right)}{p^{2}-p_{0}^{2}} \cdot \frac{\sinh \beta z}{\beta} \\
& +\frac{2 i p D\left(2 p_{0}^{2}-k^{2}\right)}{p^{2}-p_{0}^{2}}\left(e^{-\beta} 0^{z}-e^{-\beta z}\right)+4 i p\left[q\left(2 p^{2}-k^{2}\right) \delta G(p)\right. \\
& \left.+D\left(2 p^{2}-k^{2}\right)\left(2 p_{0}^{2}-k^{2}\right)+2 D k^{2} \beta_{0} \delta\right] e^{-\beta z}
\end{aligned}
$$

The pole at $\mathrm{p}=\mathrm{p}_{0}$ contributes

$$
\phi_{1}(\mathrm{x}, \mathrm{z})=-\frac{4 \mathrm{p}_{0} \delta_{0}}{\mathrm{f}^{\prime}\left(\mathrm{p}_{0}\right)}\left[\mathrm{q}\left(2 \mathrm{p}_{0}^{2}-\mathrm{k}^{2}\right) \mathrm{G}\left(\mathrm{p}_{0}\right)+2 \mathrm{D} \beta_{0}\left(2 \mathrm{p}_{0}^{2}+\mathrm{k}^{2}\right)\right] \mathrm{e}^{-i \mathrm{p}_{0} \mathrm{x}} \mathrm{e}^{-\beta_{0} \mathrm{z}}
$$

This represents the reflected wave in the region $x<0$. This does not depend upon the width $a$ of the mountain. The reflected shear wave in the region is found to be

$$
\psi_{1}(\mathrm{x}, \mathrm{z})=\frac{4 \mathrm{p}_{0} \beta_{0}}{\mathrm{f}^{\prime}\left(\mathrm{p}_{0}\right)}\left[\left(2 \mathrm{p}_{0}^{2}-\mathrm{k}^{2}\right) \overline{\mathrm{q}} \mathrm{F}\left(\mathrm{p}_{0}\right)-4 i \mathrm{p}_{0} \beta_{0} \mathrm{D}\left(2 \mathrm{p}_{0}^{2}-\mathrm{k}^{2} \beta_{0} \delta_{0}\right)\right] \mathrm{e}^{-i \mathrm{p}_{0} \mathrm{x}} \mathrm{e}^{-\delta \delta_{0} z}
$$

The integrand in (5.9) has branch points at $\mathrm{p}=\overline{\mathrm{k}}$ and $\mathrm{p}=\mathrm{k}$. The branch cuts are given by the conditions $\operatorname{Re}(\beta)=0=\operatorname{Re}(\delta)$. As discussed by Ewing and Press (1957), the parts of branch cuts are hyperbolic as shown in figure 2. For contribution along the branch cut we put $p=\bar{k}+i u, u$ being small as the main contribution is around the branch point. Along the cut, $\operatorname{Re}(\beta)=0$ and $\operatorname{Im}(\beta)$ changes signs along two sides of the cut. Since $\beta$ is imaginary, $\beta^{2}$ is negative. Therefore

$$
\beta^{2}=(\bar{k}+i u)^{2}-(\bar{k})^{2}=2 i u\left(\bar{k}_{1}+i \bar{k}_{2}\right)-u^{2}
$$

From here

$$
\beta= \pm \sqrt{\left(2 \overline{\mathrm{k}}_{2} \mathrm{u}\right)}= \pm 1 \bar{\beta}, \quad \overline{\mathrm{k}}_{1}=0
$$

Integrating (5.3) along two sides of the branch cut, we find

$$
\begin{aligned}
\phi_{2}(x, z)= & \frac{1 e^{\bar{k}_{2} x}}{2 \pi} \int_{0}^{\infty}\left[\left[\bar{\phi}_{-}(p, z)-\bar{\phi}_{-}(-p, z)\right] \beta=i \bar{\beta}\right. \\
& -\left[\bar{\phi}_{-}(p, z)-\bar{\phi}_{-}(-p, z)\right] \beta=-i \bar{\beta}^{-u x} d u \\
= & \frac{2 e^{-\bar{k}_{2} x}}{\pi} \int_{0}^{\infty}\left[H_{1}(u) \sin z \sqrt{2 \bar{k}_{2} u}+H_{2}(u) \sqrt{u} \cos z \sqrt{2 \bar{k}_{2} u}\right] e^{-u x} d u(5.14)
\end{aligned}
$$


$u$ is sma11, $H_{1}(u)$ and $H_{2}(u)$ are expanded around $u=0$ and only $H_{1}(0)$ and $H_{2}(0)$ are retained. The following Laplace integrals (Oberhettinger, (1973)) are used

$$
\begin{aligned}
& \int_{0}^{\infty} \sin a \sqrt{t} e^{-p t} d t=a \sqrt{\pi} \exp \left(-a^{2} / 4 p\right) / 2 p^{3 / 2} \\
& \int_{0}^{\infty} \sqrt{t} \cos a \sqrt{t} e^{-p t} d t=\sqrt{\pi}\left(p / 2-a^{2} / 4\right) \exp \left(-a^{2} / 4 p\right) / p^{5 / 2}
\end{aligned}
$$

The scattered waves for the region $\mathrm{x}<0$ are obtained to be

$$
\begin{aligned}
\phi_{2}(x, z)= & \frac{\left(2 \bar{k}_{2}\right)^{3 / 2}}{\sqrt{\pi}}\left[\left(2\left(\bar{k}_{2}\right)^{2}+k^{2}\right) \frac{z}{x^{3 / 2}}+2\left(\bar{k}_{2}\right)^{2}\left(\bar{k}_{2} j^{2}+k^{2}\right) .\right. \\
& \left.\frac{\left(x+\bar{k}_{2} z^{2}\right)}{x^{5 / 2}}\right]\left[q G(\bar{k})\left(2\left(\bar{k}_{2}\right)^{2}+k^{2}\right) \sqrt{\left(\left(\bar{k}_{2}\right)^{2}+k^{2}\right)}\right. \\
& \left.-2 \beta 0_{0} D k^{2} \sqrt{\left(\left(\bar{k}_{2}\right)^{2}+k^{2}\right)}-i D\left(2 p_{0}^{2}-k^{2}\right)\left(z\left(\bar{k}_{2}\right)^{2}+k^{2}\right)\right] . \\
& \exp \left[\bar{k}_{2}\left(x+z^{2} / 2 x\right)\right]
\end{aligned}
$$

when $x \gg z$, then

$$
r=\sqrt{\left(x^{2}+z^{2}\right)}=x+z^{2} / 2 x
$$

and the scattered wave is of the form

$$
\phi_{2}(x, z) \hookrightarrow \exp \left(\bar{k}_{2} r\right) / \sqrt{r}
$$

This represents a cylindrical wave. On the free surface $(z=0)$, the wave has the behaviour of $\exp \left(k_{2} x\right) / x^{3 / 2}$.

We now find the waves transmitted to the other side of the mountain. The potential for the region $x>a$ is given by

$$
\phi(x, z)=\frac{1}{2 \pi} \int_{-\infty+i \alpha_{0}^{\circ}}^{\infty+i \alpha} \bar{\phi}_{+a}(p, z) e^{-i p x} d p
$$

The countour is in the lower half of the complex plane in which $\bar{\phi}_{+a}(-p, z)$ is analytic and so

$$
0=\frac{1}{2 \pi} \int_{-\infty+i \alpha_{0}^{\circ}}^{\infty+i \alpha} \bar{\phi}_{+a}(-p, z) e^{2 i p a} e^{-i p x} d p
$$

From $(5.20)$ and $(5.21)$, we obtain

$$
\phi(x, z)=\frac{1}{2 \pi} \int_{-\infty+i \alpha_{0}^{0}}^{\infty+i \alpha}\left[\bar{\phi}_{+a}(p, z)-e^{2 i p a} \bar{\phi}_{+a}(-p, z)\right] e^{-i p x} d p
$$

We take the Fourier transform of the wave equation (2.3) from $x=a$ to $x=\infty$ to find out

$$
\mathrm{d}^{2} \bar{\phi}_{+\mathrm{a}} / \mathrm{d} \mathrm{z}^{2}-\beta^{2} \bar{\phi}_{+\mathrm{a}}=(\partial \phi / \partial \mathrm{x})_{\mathrm{a}} \mathrm{e}^{i p a}-i_{\mathrm{p}(\phi)_{\mathrm{a}}} \mathrm{e}^{i p a}
$$

Changing $p$ to $-p$ and subtracting the resulting equation from (5.23), it is found that

$$
\begin{gathered}
\left(d^{2} / d z^{2}-\beta^{2}\right)\left[\bar{\phi}_{+a}(p, z) e^{-i p a}-\bar{\phi}_{+a}(-p, z) e^{i p a}\right] \\
=2 i p D\left(2 p^{2}-k^{2}\right) e^{i p 0^{a}} e^{-\beta 0^{z}}
\end{gathered}
$$

and its complete solution is 


$$
\begin{aligned}
& \bar{\phi}_{+a}(p, z)-e^{2 i p a} \bar{\phi}_{+a}(-p, z)=D_{1}(p) e^{\beta z}+D_{2}(p) e^{-\beta z} \\
& -\frac{21 p D\left(2 p_{0}^{2}-k^{2}\right) e^{i\left(p+p_{0}\right) a} e^{-\beta} 0^{z}}{p^{2}-p_{0}^{2}}
\end{aligned}
$$

Using (4.22) and (4.23) and the procedure of (5.6), we find

$$
\begin{aligned}
\bar{\phi}_{+a}(p, z)-e^{2 i p a} \bar{\phi}_{+a}(-p, z) & =2 D_{1} \sinh \beta z-\frac{2 i p D\left(2 p_{0}^{2}-k^{2}\right) e^{i\left(p+p_{0}\right) a} e^{-\beta} z}{p^{2}-p_{0}^{2}} \\
& +g(p) \exp (-\beta z) / f(p)
\end{aligned}
$$

where

$$
\begin{aligned}
g(p) & =+2 i p\left[-\left(2 p^{2}-k^{2}\right) q \delta G(p)\left(1+e^{2 i p a}\right)-2 i p \beta \delta \bar{q} F(p)\left(e^{2 i p a}-1\right)\right. \\
& +2 p \beta \delta D\left(2 p_{0}^{2}-k^{2}\right)\left(\frac{1}{p+p_{0}}+\frac{e^{2 i p a}}{p-p_{0}}\right) \\
& +2 p_{0}^{\beta} 0^{\delta D}\left(2 p^{2}-k^{2}\right)\left(\frac{1}{p+p_{0}}-\frac{e^{2 i p a}}{p-p_{0}}\right) \\
& -2 p\left(2 p_{0}^{2}-k^{2}\right) \beta \delta D\left(\frac{1}{p+p_{0}}+\frac{e^{2 i p a}}{p-p_{0}}\right) \\
& -2\left(2 p^{2}-k^{2} p_{0} p_{0} \delta D D\left(\frac{1}{p+p_{0}}-\frac{e^{2 i p a}}{p-p_{0}}\right)\right]
\end{aligned}
$$

$D_{1}$ in (5.26) is eliminated by the procedure for $E$ in (5.7). It is obtained that

$$
\begin{aligned}
& \bar{\phi}_{+a}(p, z)-e^{2 i p a} \bar{\phi}_{+a}(-p, z)=\frac{\sinh \beta z}{\beta}\left[\beta \overline{q F}(p)\left(1-e^{2 i p a}\right)\right. \\
&\left.+i D\left(2 p_{0}^{2}-k^{2}\right)(\beta-\beta)\left(\frac{1}{p+p_{0}}+\frac{e^{2 i p a}}{p-p_{0}}\right)\right] \\
&-21 p D\left(2 p_{0}^{2}-k^{2}\right) e^{i\left(p+p_{0}\right) a} e^{-\beta 0^{z}} /\left(p^{2}-p_{0}^{2}\right) \\
&+g(p) \exp (-\beta z) / f(p)
\end{aligned}
$$

The pole $p=-p_{0}$ in the integrand in $(5.22)$ contributes

$$
\begin{aligned}
\phi_{3}(x, z)= & -D\left(2 p_{0}^{2}-k^{2}\right) e^{i p_{0} x} e^{-\beta} 0^{z}-4 p_{0}\left[q \delta \delta_{0}\left(2 p_{0}^{2}-k^{2}\right) G\left(-p_{0}\right) \cos 2 p_{0} a\right. \\
& \left.-2 p_{0} 0_{0} \delta 0 q F\left(-p_{0}\right) \sin 2 p_{0} a\right] e^{i p_{0}(x-a)} e^{-\beta_{0} z} / f^{\prime}\left(-p_{0}\right)
\end{aligned}
$$

The first term cancels exactly the incident wave and the second term is the transitional wave in the region $x>a$.

6. CONCLUSIONS.

The integrals along the branch cuts.at $p=-\bar{k}, \pm_{k}$ lead to the scattered waves as obtained in (5.19). They behave as $\exp \left(-\bar{k}_{2} r\right) / \sqrt{r}$, where $r$ is the distance from the scatterer. These are cylindrical waves which die at distant points from the foot of the mountain. On the free surface $(z=0)$, the scattered waves have the form $\exp \left(-\bar{k}_{2} x\right) / x^{3 / 2}$ which is large at the points near the scatterer. Thus the energy of the scattered waves is very large close to the scatterer and diminishes as the wave moves away from it. The 
transmitted wave in (5.29) depends upon width a of the mountain. As the distance from the other end of the mountain increases, the transmitted wave decreases exponentially and dies out at distant points. The reflected waves are given by (5.10) and (5.11) which do not depend upon the width of the mountain. Numerical results for the amplitude of the scattered wave have been computed for Poisson's solids for which $k=\sqrt{3} k$ at a point $(r=1 / 2 \mathrm{~km}, z=0)$ in the region $\dot{x}<0$ of the free surface. The results are obtained for $q=0$ and $B=1.8932 \bar{k}$. There is a sharp increase in the amplitude (fig. 3) when the wave number $\bar{k}$ increases through small values.

The results of this paper have their application to underground nuclear explosions carried out on either side of the mountains like Himalayas. It helps calculating the amount of energy reflected and scattered at the foot of the mountain and the amount of energy which is transmitted to the other side of the mountain.

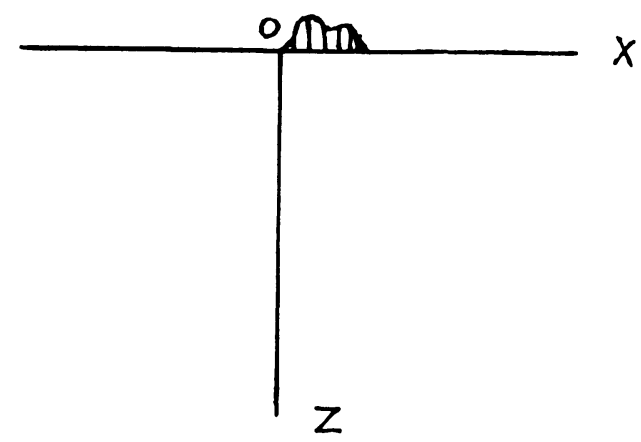

PIG. 1

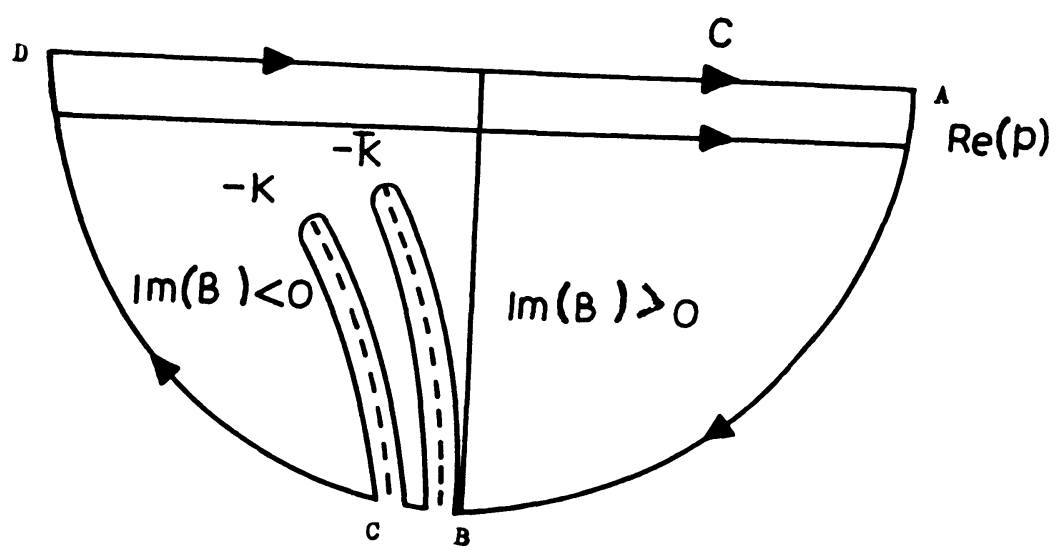

FIG. 2 


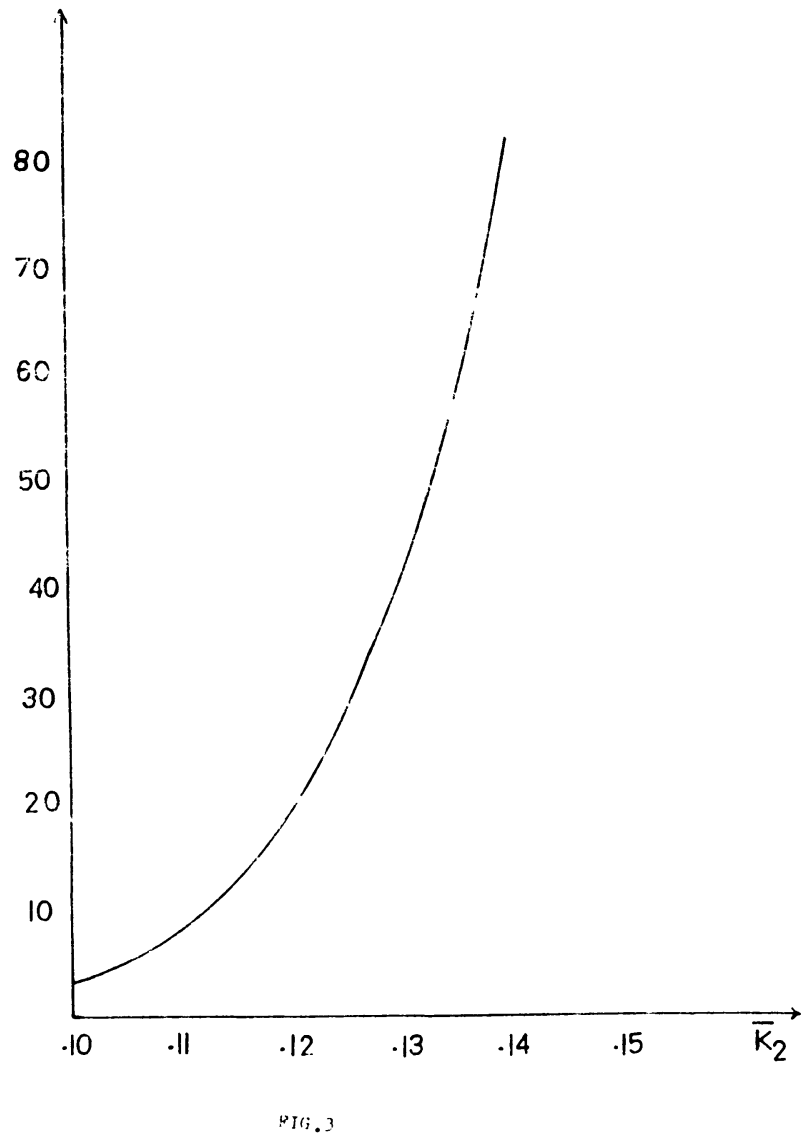

\section{REFERENCES}

1. DESHWAL, P. S. Diffraction of a Compressional Wave by a Rigid Barrier in a Liquid Half-space, Pure App1. Geophys., 85(1971), 107-124.

2. DESHWAL, P. S. Diffraction of Compressional waves by a Strip in a Liquid Half-space, Jr. Math. Phys. Sci., 15(1981), 263-281.

3. EWING, W. M., JARDETSKY, W. S., and PRESS, F. Elastic Waves in Layered Media, McGraw Hill Book Co., 1957.

4. KAZI, M. H. The Lovewave Scattering Matrix for a Continental Margin, Geophys. J. R. Ast. Soc., 52(1978), 25-44.

5. MOMOI, T. Scattering of the Rayleigh Wave by a Semi-circular Rough Surface, J. Phys. Earth, 28 (1980), 497-519.

6. MOMOI, T. Scattering of Rayleigh Waves by a Rectangular Rough Surface, J. Phys. Earth, 30 (1982), 295-319.

7. NOBLE, B. Methods Based on the Wiener-Hopf Technique, 1958.

8. OBERHETTINGER, F. and BADII, L. Tables of Laplace transforms, Springer-Verlag, New York, 1973.

9. SATO, R. Love Waves in Case the Surface layer is Variable in Thickness, J. Phys. Earth, 9 (1961), 19-36. 


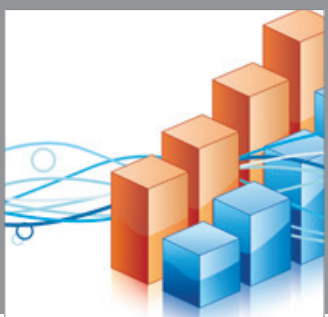

Advances in

Operations Research

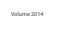

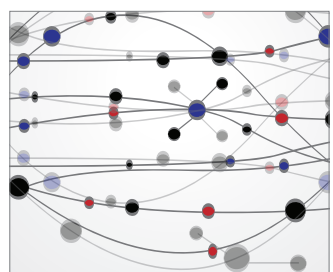

\section{The Scientific} World Journal
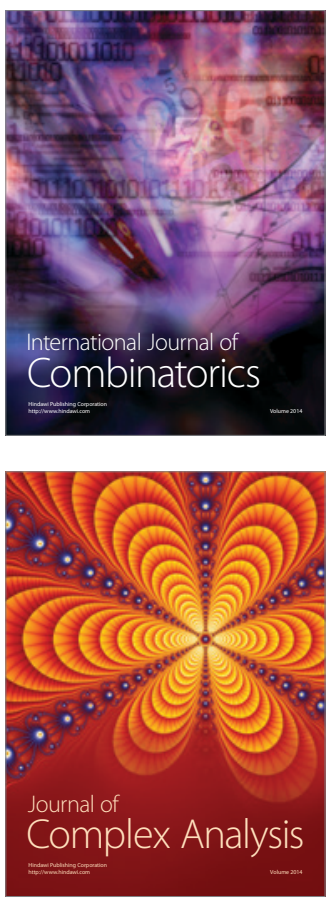

International Journal of

Mathematics and

Mathematical

Sciences
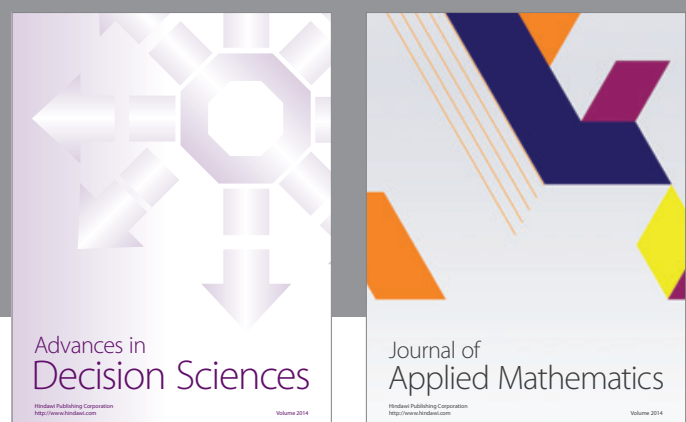

Journal of

Applied Mathematics
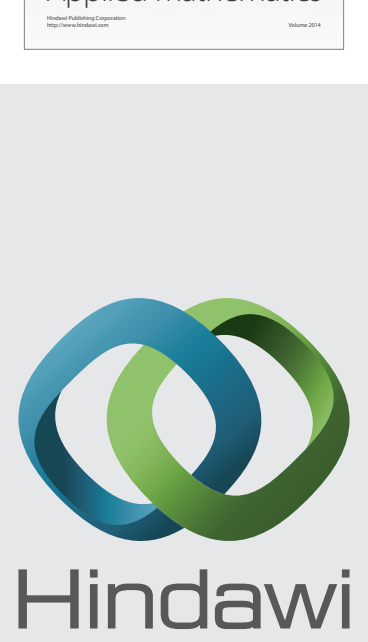

Submit your manuscripts at http://www.hindawi.com
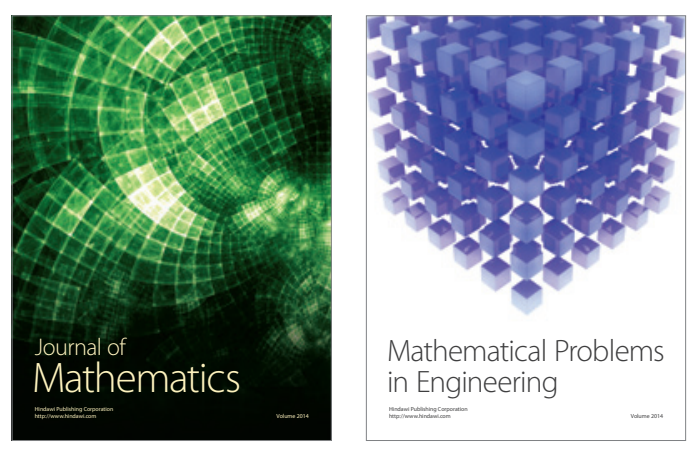

Mathematical Problems in Engineering
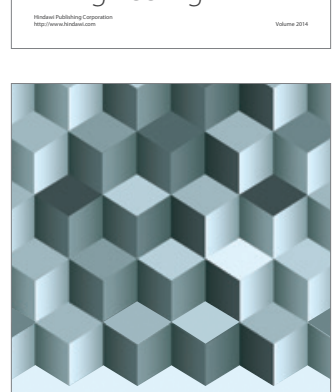

Journal of

Function Spaces
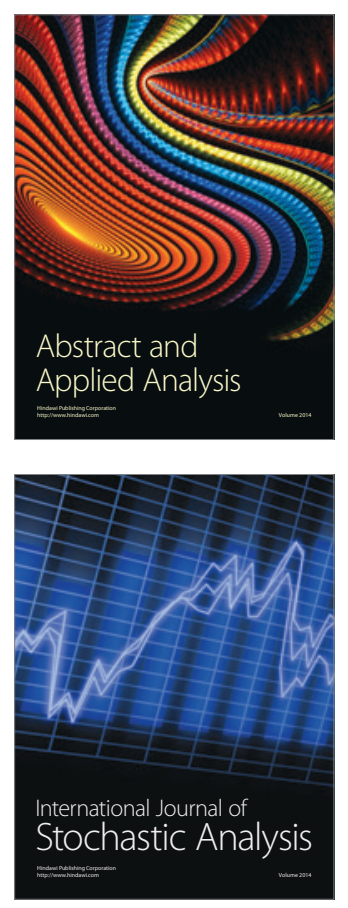

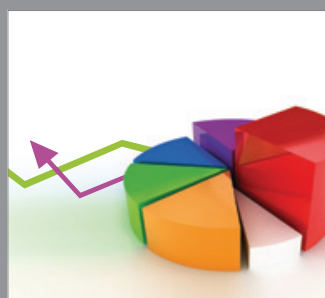

ournal of

Probability and Statistics

Promensencen
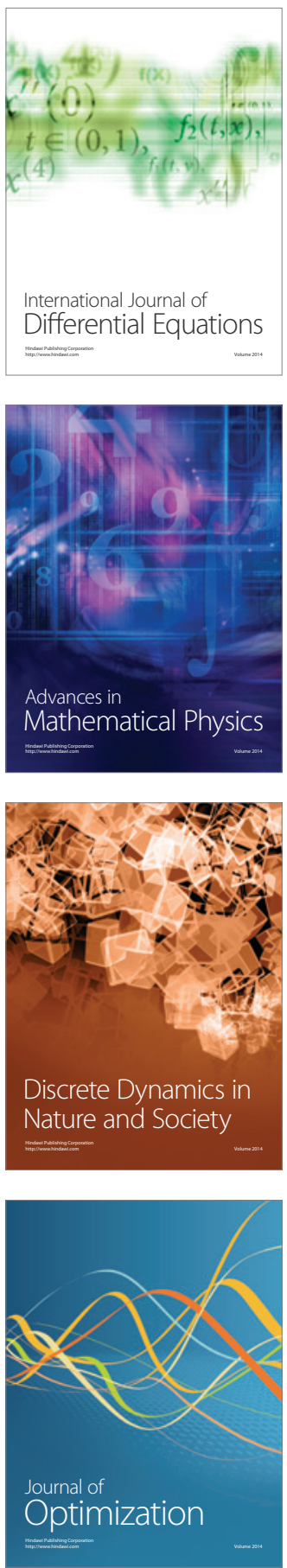